\title{
Differenz in der Einheit: Ein ostdeutscher Sonderweg im betrieblichen Gesundheitsschutz
}

Karina Becker/Ulrich Brinkmann/Thomas Engel

Zwischen gesetzlich formuliertem Anspruch und betriebspraktischer Wirklichkeit im deutschen Arbeits- und Gesundheitsschutz klafft eine Lücke. Für Ostdeutschland stellt sich dieses Defizit noch deutlicher dar, wie wir anhand verschiedener empirischer Befunde aus Beschäftigten- und Betriebsratsbefragungen zeigen. Im Licht der Erfahrungen aus DDR-, Transformations- und Konsolidierungszeit diskutieren wir die Voraussetzungen für eine gezielte Gesundheitsschutzpolitik: Durch die strategische Beteiligung von Beschäftigten und ihrer Interessenvertretungen kann der gesetzliche Anspruch betriebliche Wirklichkeit werden.

\section{Diskrepanz zwischen Anspruch und Wirklichkeit als Normalfall}

Mit dem Ausklingen der Transformationsforschung ist es etwas aus der Mode gekommen, sich mit Ost-West-Unterschieden in Deutschland zu beschäftigen. Die Jubiläumsrückschau auf 20 Jahre deutsche Einheit indes wird diesen Aspekt wieder stärker ins öffentliche Bewusstsein rücken. In den nächsten Monaten wird allenthalben Bilanz gezogen, und damit kommen die Entwicklungspfade der zwei Landesteile in den Fokus.

Die Debatten um den sozioökonomischen Angleichungsprozess werden in der Regel bestimmt vom Verweis auf die Produktivitätslücke zwischen Ost und West und die Lohndifferenz, wobei Letzteres teilweise auf Ersteres zurückgeführt wird. Diese zwei quantitativ bestimmbaren Kennziffern verharren in der Ost-WestDifferenz nach einer langen Aufholphase inzwischen auf zweistelligem Prozentpunkteniveau. ${ }^{1}$ Ostdeutschland bleibt bei Wirtschaftsleistung, Arbeitsplatz- und Lohnbilanz weiter im Rückstand.

Wie sieht der Vergleich im Arbeits- und Gesundheitsschutz (AGS) aus - einem Ausschnitt betrieblicher Arbeitspolitik, der Auskunft über qualitative Angleichungsund divergierende Entwicklungsprozesse gibt. Auf den ersten Blick scheinen sich AGS in Ost und West nicht mehr voneinander zu unterscheiden: Für beide Teile gelten die gleichen gesetzlichen Standards (Arbeitsschutzgesetz, Arbeitssicherheitsgesetz, Betriebsverfassungsgesetz etc.) und institutionellen Rahmenbedingungen. Sie werden durch bundesweit ähnlich organisierte überbetriebliche Institutionen - dem dualen System aus Berufsgenossenschaften und Gewerbeaufsichtsämtern - getragen und durch vergleichbare betriebliche Akteurskonstellationen in diesem Feld geprägt (Becker et al. 2010a). Dementsprechend differenzieren Studien und Statistiken zu diesem Thema kaum noch zwischen Ost und West und führen Unterschiede eher auf regionale Besonderheiten oder Branchenunterschiede zurück.

Wir gehen jedoch davon aus, dass die jeweiligen gesellschaftlichen Rahmenbedingungen bis heute auch im AGS ihre Spuren hinterlassen haben. So wirken einige für die DDR typische Betriebskulturen und Varianten innerbetrieblicher Verarbeitung organisationsexterner Anforderungen bis heute nach. Der Transformationsprozess forderte den Akteuren in kürzester Zeit eine Integration und Adaption von Marktprozessen und Wettbewerbsbedingungen sowie die Aneignung neuer gesetzlicher Vorgaben und institutioneller Rahmensetzung ab (Fröhner 1997, S. 24). Sie haben dazu beigetragen, dass bis heute eine Lücke zwischen Anspruch und Wirklichkeit erhalten blieb, wobei sich das Ausmaß dieser Kluft nicht wesentlich verändert hat, wohl aber die Brücken, mit denen eine Überwindung versucht wurde (Brinkmann 2002; Offe 1994).

Probleme zwischen den gesetzlichen AGS-Vorgaben und der Umsetzung im Betrieb gibt es freilich auch in Westdeutschland. Gerade die Neuerungen im Arbeitsschutzgesetz 1996 und die folgende Rechtsprechung machen immer wieder offenkundig, dass diese Diskrepanz der
Normalfall ist, wie häufig am Beispiel der Verbreitung der Gefährdungsbeurteilung gezeigt wird (Beck/Lenhardt 2009). So führen fehlende oder durchsetzungsschwache Interessenvertretungen in den Betrieben dazu, dass Gefährdungsbeurteilungen nicht oder nur unzureichend durchgeführt werden. Dabei zeigen viele Beispiele, dass

\footnotetext{
„Je Erwerbstätigem werden weniger als $80 \%$ der westdeutschen Wirtschaftsleistung erbracht " (Brenke/Zimmermann 2009, S. 15). Aufgrund längerer Arbeitszeiten „.... ist der Abstand bei der Stundenproduktivität noch größer. Je Erwerbstätigenstunde waren es 2008 nur $74 \%$ der westdeutschen Wertschöpfung" (ebd.). 2002 erreichten die ostdeutschen Tariflöhne knapp $93 \%$ des Westniveaus, das Effektivlohnniveau lag mit $81 \%$ deutlich darunter (Görzig et al. 2005, S. 336).
}

Karina Becker, Dr., Soziologin, wissenschaft-
liche Mitarbeiterin, Bereich Arbeits-,
Industrie- und Wirtschaftssoziologie der
Friedrich-Schiller-Universität Jena. Arbeits-
schwerpunkte: Finanzmarktkapitalismus,
KMU, betriebliche Mitbestimmung,
Arbeits- und Gesundheitsschutz.
e-mail: karina.becker@uni-jena.de
Ulrich Brinkmann, Prof. Dr., lehrt Wirt-
schaftssoziologie an der Universität Trier,
Arbeitsschwerpunkte: Organisations-
soziologie, Betrieblicher Arbeits- und
Gesundheitsschutz, Gewerkschaften,
Beteiligungsforschung.
e-mail: brinkman@uni-trier.de
Thomas Engel, Soziologe, wissenschaftlicher
Mitarbeiter, Bereich Arbeits-, Industrie- und
Wirtschaftssoziologie der Universität Jena.
Arbeitsschwerpunkte: Betrieblicher Arbeits-
und Gesundheitsschutz, Mitbestimmung,
demografischer Wandel.
e-mail: thomas.engel@uni-jena.de


die Beteiligung von Betriebsräten (aber auch von Beschäftigten direkt) entscheidende Impulse für die Verbesserung der Qualität von Gefährdungsbeurteilungen setzen kann. Dieser Zusammenhang wurde kürzlich erneut für die Berücksichtigung psychischer Belastungen nachgewiesen (Lenhardt et al. 2010, S. 461f.) sowie für eine verbesserte Dokumentation und Maßnahmenableitungspraxis in der Gefährdungsbeurteilung (vgl. Ahlers/Brussig 2005).

Die geringe Größe der Betriebe erschwert vielerorts die Etablierung eines wirksamen und nachhaltigen Gesundheitsmanagements. Zudem setzen die überbetrieblichen Einrichtungen durch die risikobedingte Tarifeinordnung der Betriebe - hier wie dort - Anreize für eine Verschleierung von nicht-meldepflichtigen Unfällen oder Beinaheunfällen, aus denen vielfache Erkenntnisse für Verbesserungen gezogen werden könnten. Neben all diesen Erklärungsansätzen, die auch den Ostteil des Landes kennzeichnen, nehmen wir darüber hinaus dessen kulturell eingebetteten Entwicklungspfad in den Blick, der - wie sich zeigen wird - noch heute den ostdeutschen Weg prägt.

Die Kernthese ist, dass das generelle Problem einer beständigen Diskrepanz zwischen gesetzlich formuliertem Anspruch und betriebspraktischer Wirklichkeit im deutschen AGS-System in Ostdeutschland ausgeprägter ist (Abschnitt 2). Dies wird durch einen Methodenmix aus Beschäftigten- und Betriebsratsbefragungen verschiedener sozialwissenschaftlicher Institute sowie die betriebspraktischen Erfahrungen aus dem PARGEMA-Projekt herausgearbeitet. Dieser Analyse liegt die Hypothese zugrunde, dass die Ursachen für die größere Diskrepanz auch in der besonderen Entwicklung ausgehend vom AGSSystem der DDR über die Transformationszeit bis hin zu einer anschließenden wirtschaftlichen Konsolidierungsphase zu suchen sind (Abschnitt 3). Zur Kennzeichnung des ostdeutschen Sonderweges werden die betrieblichen Sozialbeziehungen, die machtpolitischen Voraussetzungen für Interessenhandeln und die Beteiligungsspielräume für die Beschäftigten diskutiert (Abschnitt 4). Daran anknüpfend werden abschließend Voraussetzungen für eine gezielte Gesundheitsschutzpolitik durch die strategische Beteiligung von Beschäftigten und ihrer Interessenvertretungen formuliert (Abschnitt 5).

\section{Differenz in der Einheit: Belastungssituation und Gesundheitsschutz}

\subsection{AUSGEPRÄGTE BELASTUNGS- UND GEFÄHRDUNGSLAGEN}

Bei der Betrachtung von Unterschieden in der täglichen Praxis des betrieblichen AGS kommt man nicht umhin, die Arbeitsbedingungen in den beiden Landesteilen unter die Lupe zu nehmen. Sie sind einerseits Auslöser und Antrieb für bestimmte AGSAktivitäten, andererseits sind sie auch Ausdruck einer spezifischen AGS-Qualität. Ursache und Wirkung sind nicht immer eindeutig bestimmbar. Unterschiedliche Belastungs- und Gefährdungslagen der Beschäftigten in den ostdeutschen Betrieben stehen in Zusammenhang mit der Branchenstruktur und den überbetrieblich wie betrieblich getroffenen expliziten und impliziten Vereinbarungen. Als Beispiel für eine solche explizite Verständigung sei auf die Tarifvertragsgestaltung jenseits der Entlohnungsbedingungen, z. B. zur Arbeitszeit, verwiesen, die trotz schwacher Tarifbindung in den ostdeutschen Ländern eine gewisse Normierung nach sich zieht.

Hinsichtlich der Arbeitsbedingungen lässt sich anhand der Daten aus der $\mathrm{BiBB} / \mathrm{BAuA}$-Erwerbstätigenbefragung 2005/2006 ( $n=20.000)$ ein Tätigkeitsund Belastungsprofil erstellen. ${ }^{2}$ So sind die zu erledigenden Arbeitsaufgaben in Ostdeutschland häufiger durch Arbeit im Stehen, durch klimatisch schwierige Bedingungen (Kälte, Hitze, Nässe, Zugluft) und durch Heben und Tragen geprägt, während zu den Tätigkeiten in westdeutschen Unternehmen mit höherer Wahrscheinlichkeit Arbeit im Sitzen, Arbeiten mit Schutzausrüstung oder -kleidung gehören (Tabelle 1).

Durch die Frage der Belastungswahrnehmung wird das Bild der Differenzen vielschichtiger. So signalisieren Erwerbstätige in Ostdeutschland häufiger als die Befragten der Vergleichsgruppe, dass die Beleuchtungsbedingungen sowie das Rauchen am Arbeitsplatz belastend wirken. Hinsichtlich der Arbeitsanforderungen, die auch psychische Fehlbelastungen hervorrufen können, werden ebenfalls Unterschiede offenkundig: Im Relativvergleich absolvieren die Beschäftigten in Ostdeutschland häufiger als in Westdeutschland detailliert vorgeschriebene, monotone Abläufe mit Zielvorgaben in Form von Stückzahlen, Mindest- oder Zeitleistungsnormen. Diese scheinbar stärker tayloristisch geprägten Abläufe enthalten aber im Absolutvergleich die gleichen Ausmaße von Anforderungen wie sie im westdeutschen Produktionssystem gängig sind: gleichzeitige Überwachung verschiedenartiger Arbeiten und Vorgänge oder hoher Termin- und Leistungsdruck. Dort betonen die Erwerbstätigen wiederum vergleichsweise überdurchschnittlich häufig die Notwendigkeit der Einarbeitung in neue Aufgaben sowie Arbeitsstörungen und -unterbrechungen.

Die Wahrnehmung psychischer Belastungen wird insgesamt von ostdeutschen Befragten stärker betont. Sie hat gegenüber den westdeutschen Betrieben überdurchschnittlich oft mit einer empfundenen Verantwortung für die finanziellen Verluste, die durch Fehler entstehen können, zu tun. Auch häufige Arbeitsstörungen werden von den Ostdeutschen als überdurchschnittlich belastend empfunden. Das Arbeiten bis an die Belastungsgrenze wird dagegen von der Mehrheit aller Befragten als problematisch bewertet.

Psychische Belastungen ergeben sich jedoch nicht alleine aus den Anforderungen der konkreten Arbeitsaufgaben. Das Gratifikationskrisenmodell geht von einem starken Einfluss solcher Faktoren auf die Gesundheit aus, die Anerkennung für die Beschäftigten und ihre Arbeitsleistung voraussetzen. Dazu gehören die Entlohnung der Leistung genauso wie nichtmonetäre Anerkennung oder die Wertschätzung in Form von Entwicklungschancen durch Aufstieg oder Weiterbildung (Siegrist/Dragano 2008).

Kursorisch sei an dieser Stelle nur auf die weitgehend bekannten Einkommensund Arbeitszeitdifferenzen hingewiesen. So liegt der Median des Bruttoverdienstes der durch die BiBB-/BAuA-Befragung erfassten Erwerbstätigen im Osten bei $2.000 €$, im Westen bei $2.500 €$. Die vereinbarten Wochenarbeitszeiten divergieren dabei sehr stark: Während sich $38 \%$ der Beschäftigten im Westen zwischen

2 Unser besonderer Dank gilt der Bundesanstalt für Arbeitsschutz und Arbeitsmedizin, namentlich Michael Ertel, der uns für die Auswertung der Daten der BIBB/BAuA-Erwerbstätigenbefragung 2005/2006 wichtige Hinweise gab. 
Tabelle 1: Differenzen zwischen Ost- und Westdeutschland bei Tätigkeitsmerkmalen und

Belastungswahrnehmung - in \% -

\begin{tabular}{|c|c|c|c|c|c|c|}
\hline & \multicolumn{3}{|c|}{ Häufiges Arbeiten ... } & \multicolumn{3}{|c|}{ Belastungswahrnehmung von Arbeiten ... } \\
\hline & West & Ost & Differenz & West & Ost & Differenz \\
\hline \multicolumn{7}{|l|}{ Physisch wirksame Tätigkeitsmerkmale } \\
\hline im Stehen & 55,5 & 61,9 & 6,4 & 21,2 & 23,8 & 2,6 \\
\hline unter Kälte, Hitze, Nässe, Feuchtigkeit, Zugluft & 20,5 & 25,2 & 4,7 & 48,0 & 47,0 & $-0,9$ \\
\hline mit Heben und Tragen ${ }^{1)}$ & 22,2 & 26,9 & $4,7^{*}$ & 43,3 & 42,2 & $-1,2$ \\
\hline bei grellem Licht oder schlechter Beleuchtung & 9,1 & 9,9 & 0,8 & 47,2 & 53,2 & $6,0^{*}$ \\
\hline an einem Platz, an dem geraucht wird & 17,3 & 14,9 & $-2,3$ & 27,9 & 31,4 & $3,5^{*}$ \\
\hline mit Schutzkleidung oder Schutzausrüstung & 21,9 & 18,3 & $-3,6^{*}$ & 11,3 & 12,6 & 1,2 \\
\hline im Sitzen & 54,4 & 47,4 & $-7,0^{*}$ & 16,2 & 16,9 & 0,7 \\
\hline \multicolumn{7}{|l|}{ Psychisch wirksame Tätigkeitsmerkmale } \\
\hline mit sich bis in alle Einzelheiten wiederholendem Arbeitsgang & 50,6 & 55,7 & $5,1^{*}$ & 12,9 & 11,6 & $-1,4$ \\
\hline mit bis ins Einzelne vorgeschriebener Arbeitsdurchführung & 22,3 & 26,9 & $4,6^{*}$ & 25,5 & 26,0 & 0,5 \\
\hline $\begin{array}{l}\text { mit vorgeschriebenen genauen Stückzahlen, bestimmten } \\
\text { Mindestleistungen oder Zeitvorgaben }\end{array}$ & 30,6 & 33,9 & $3,3^{*}$ & 38,1 & 39,1 & 1,0 \\
\hline $\begin{array}{l}\text { mit größeren finanziellen Verlusten bei kleinen Fehlern bzw. } \\
\text { geringer Unaufmerksamkeit }\end{array}$ & 15,8 & 14,2 & $-1,6$ & 48,1 & 57,1 & $9,0^{*}$ \\
\hline $\begin{array}{l}\text { mit neuen Aufgaben, in die man sich erst hineindenken/ } \\
\text { einarbeiten muss }\end{array}$ & 39,5 & 36,4 & $-3,1^{*}$ & 12,7 & 10,5 & $-2,2$ \\
\hline mit Störungen und Unterbrechungen & 47,5 & 39,2 & $-8,4^{*}$ & 48,6 & 54,8 & $6,2^{*}$ \\
\hline \multicolumn{7}{|c|}{$\begin{array}{l}\text { 1) von Lasten mehr als } 20 \mathrm{~kg} \text { (Männer) bzw. } 10 \mathrm{~kg} \text { (Frauen). } \\
\text { ”Für diese Tabelle wurden Chi-Quadrat-Tests durchgeführt. Zur besseren Lesbarkeit wurden hier nur die Prozent-Differenzen dargestellt und die signifikanten Unterschiede ( } \mathrm{p}<0,05) \\
\text { mit einem * gekennzeichnet. } \\
\text { Quelle: BiBB/BAuA-Erwerbstätigenbefraqung 2006. Berechungen der Autoren ohne Berlin. }\end{array}$} \\
\hline
\end{tabular}

35 und unter 40 Stunden bewegen und weitere $28 \% 40$ Stunden und mehr arbeiten, sind es im Osten nur $17 \%$ bzw. sogar $53 \%$. D. h. hier besteht allein beim Verhältnis Lohn-/Gehaltshöhe zu aufgewendeter Arbeitszeit eine erhebliche Diskrepanz zu Westdeutschland.

Verschlechtert sich die betriebliche ökonomische Situation so stark, dass z.B. Standortsicherungsvereinbarungen verhandelt werden, sehen die Zugeständnisse der Beschäftigten im Osten eher Einkommenseinbußen vor, während sie sich im Westen eher auf die Arbeitszeit beziehen (Arbeitszeitverlängerung oder -flexibilisierung), wie sich mithilfe der Daten aus der PARGEMA/WSI-Betriebsrätebefragung 2008/09 zeigen lässt. Der überdurchschnittliche Einsatz der ostdeutschen Beschäftigten in Schichtarbeitszeitmodellen und in Rufbereitschaft lässt den Interessenvertretern offenkundig wenig Spielraum, sowohl bei den Standortsicherungsvereinbarungen als auch bei der Umgestaltung belastender Arbeitsbedingungen. ${ }^{3}$

In einer zusammenfassenden Darstellung von Belastungen, wie sie durch den DGB-Index „Gute Arbeit ${ }^{\text {“4 }}$ vorgenommen wird und in den auch Fragen zur Einkommenssituation und Arbeitsplatzsicherheit eingehen, kommt man demzufolge zu dem Schluss, dass knapp $39 \%$ der Beschäftigten in Ostdeutschland gegenüber $29 \%$ in
Westdeutschland Bedingungen unterhalb der durchschnittlichen Werte für Arbeitsqualität vorfinden. Gute Arbeitsbedingungen liegen den Befragten zufolge nur für $9 \%$ (Ost) bzw. $15 \%$ (West) vor. Inwieweit diese Unterschiede auch in der unterschiedlichen AGS-Praxis begründet sind, soll im Folgenden diskutiert werden.

Zur Erfassung und Kennzeichnung psychischer Belastungen lässt sich die Häufigkeit von Ausfalltagen als ein Indikator heranziehen. So weist der letzte Fehlzeitenreport starke Unterschiede zwischen den einzelnen Bundesländern auf, die sich in der Tendenz auch als Ost-West-Differenz lesen lassen. Demnach melden sich in Thüringen, Sachsen, Sachsen-Anhalt, Mecklenburg-Vorpommern und Brandenburg bis heute anteilsmäßig weniger Erwerbstätige aufgrund psychischer Belastungen krank als in den meisten westdeutschen Ländern (Heyde/Macco 2010, S. 35). Muss man deswegen zu der Schlussfolgerung kommen, dass die Arbeitsbelastungen in den östlichen Bundesländern kaum problematisch sind? Wohl kaum. Vor dem Hintergrund der dargestellten Arbeitsbedingungen aus Beschäftigten- und Betriebsräteperspektive stellt sich eher die Frage, wie es angesichts überdurchschnittlicher Belastungen $\mathrm{zu}$ unterdurchschnittlichen Fehlzeiten kommt. Dahinter vermuten wir neben der nachwirkenden „Disziplinierungskeule“ durch Personalentlassungen in der Trans- formationszeit auch den schwächeren Einfluss von Schutzmechanismen für die Beschäftigten vonseiten der Interessenvertretungen und des Arbeitsschutzes sowie eine ausgeprägte Kultur der individuellen und betrieblichen Krankheitsverleugnung (Kocyba/Voswinkel 2007).

\subsection{KONVENTIONELLE GEFÄHRDUNGSBEURTEILUNG}

Auf der Grundlage von drei Datenquellen lassen sich Aussagen zur Gefährdungsbeurteilung (GFB) treffen, dem zentralen AGS-Instrument, das nach den Vorgaben des Arbeitsschutzgesetzes ( $\$ 5$ ArbSchG) dem Zweck dient, „durch eine Beurteilung die für die Beschäftigten mit ihrer Arbeit verbundene Gefährdung zu ermitteln“.

(1) Die WSI-Betriebsrätebefragungen 2004 (Ahlers/Brussig 2005) und 2008/09 zeigen, dass dieses Instrument verlässlich insgesamt nur in $52 \%$ bzw. $46 \%$ der Betriebe ab 20 Beschäftigten angewendet wurde.

3 Die Auswertungen der WSI-Betriebsrätebefragung zu den Ost-West-Unterschieden bei den Zugeständnissen zu Standortsicherungsvereinbarungen und den Arbeitszeitregelungen können bei Interesse nachgeliefert werden.

4 In den DGB-Index-Wert gehen Beschäftigtenurteile zu Einkommen/Sicherheit, Ressourcen und Belastungen ein (vgl. Fuchs 2007; http://www. dgb-index-gute-arbeit.de). 
Die Nutzungswahrscheinlichkeit steigt mit der Betriebsgröße, was auch auf die Unterstützung durch funktionierende Mitbestimmungsstrukturen zurückzuführen ist. Notwendige Bestandteile der GFB wie die Umsetzung abgeleiteter Maßnahmen (2008/09: 62 \%; Prozentwerte beziehen sich auf GFB durchführende Unternehmen), Wirksamkeitskontrolle und Dokumentation (52\%) sowie Berücksichtigung psychischer Belastungen (29\%), sie werden jedoch in deutlich geringerem Ausmaß anspruchsgerecht erfüllt. Überraschend hoch fällt mit $52 \%$ zunächst der Anteil der GFB durchführenden Unternehmen in Ostdeutschland (West: $45 \%$ ) aus. Die Unternehmen, die durch die Befragungen dargestellt werden, sind jedoch nicht typisch für Ostdeutschland, weil sie aufgrund der geringen Betriebsratsdichte überdurchschnittlich stark die Unternehmen mit günstigen ökonomischen Perspektiven, mit Tarifbindung oder -anlehnung, mit aussagekräftigen und arbeitsfähigen Mitbestimmungsvertretern berücksichtigen.

(2) Die Erwerbstätigenbefragung des BiBB und der BAuA 2006 wirft ein anderes Licht auf die Verbreitung des Instruments durch den Fokus auf den Einzelarbeitsplatz bzw. die Arbeitskraft anstelle der betrieblichen Perspektive. Demzufolge geben $37 \%$ (West) bzw. 29 \% (Ost) der abhängig Beschäftigten an, dass eine Gefährdungsanalyse an ihrem Arbeitsplatz durchgeführt wurde. Dabei hängt der Begriff „Gefährdungsanalyse“ den Anspruch der umfassenderen GFB bereits niedriger. Wir haben es demnach im Vergleich zu den Befunden aus der WSI-Betriebsrätebefragung (bei deutlich repräsentativerem Sample) nicht nur mit einer schwächeren Nutzung des AGS-Instruments in Betrieben ohne Interessenvertretung zu tun, sondern auch mit einer eingeschränkten innerbetrieblichen Reichweite und Qualität.

Darauf verweist auch der Befund, dass auffallend häufiger westdeutsche Betriebe die Gefährdungsanalyse zur Ableitung von Maßnahmen der betrieblichen Gesundheitsförderung (BGF) nutzen. Während $56 \%$ der westdeutschen Beschäftigten, die an einer BGF teilgenommen hatten, auch über eine Gefährdungsanalyse für ihren Arbeitsplatz berichten konnten, sind es im Osten nur $48 \%$. Die Beschäftigten im Osten machen seltener die Erfahrung einer ganzheitlichen, systematischen Herangehensweise an den AGS. Daraus

Tabelle 2: Gefährdungsbeurteilungen (GFB) nach Vorhandensein einer betrieblichen Interessenvertretung - in \% der Beschäftigten -

\begin{tabular}{lcc}
\hline & $\begin{array}{c}\text { Betriebsrat/Personalvertretung } \\
\text { vorhanden }\end{array}$ & $\begin{array}{c}\text { Betriebsrat/Personalvertretung } \\
\text { nicht vorhanden }\end{array}$ \\
\hline GFB nach ArbSchG & 23,2 & 9,7 \\
konventionelle GFB & 14,7 & 6,9 \\
keine GFB & 32,6 & 53,5 \\
nicht bekannt/weiß nicht & 29,5 & 29,8 \\
Summe & 100,0 & 100,0 \\
\hline GFB nach ArbSchG - Gefährdungsbeurteilung nach Arbeitsschutzgesetz einschl. Berücksichtigung psychischer Belastungen. \\
Konventionelle GFB - konventionelle Gefährdungsbeurteilung ohne Berücksichtigung \\
psychischer Belastungen.
\end{tabular}

spricht auch eine stärkere Neigung der betrieblichen Akteure zu einem klassischen Arbeitsschutzverständnis, während in Westdeutschland z. B. häufiger auch psychische und physische Belastungen integriert betrachtet werden.

(3) Die Befragung im Rahmen des DGBIndex Gute Arbeit 2009 kann ebenfalls die Beschäftigtenperspektive klären, wartet sogar mit aktuelleren Daten auf und kann die Aussagen zur Qualität von Arbeit und der Schwere von Belastungen mit den interessenpolitischen Konstellationen im Betrieb verknüpfen. ${ }^{5}$ Als Indikator für die Qualität des Gesundheitsschutzes wird auch hier die GFB bzw. die niedrigschwelligere Gefährdungsanalyse herangezogen. Dabei wird unterschieden zwischen der Erfassung und Bewertung von Belastungen nach Arbeitsschutzgesetz (ArbSchG) - explizit durch die Formulierung „Berücksichtigung psychischer Belastungen“ abgefragt - und der konventionellen GFB (also zumeist einmalig durchgeführt, ohne Einbeziehung der Beschäftigten und ohne Erfassung psychischer Belastungen). ${ }^{6}$ Die DGB-Index-Befragung zeigt deutlich, wie stark die Qualität der GFB vom Vorhandensein einer Interessenvertretung im Betrieb abhängt (Tabelle 2).

Der Zusammenhang zwischen Qualität der Arbeit und Qualität der Gefährdungsbeurteilung ist eindeutig: Eine gute Bewertung der Arbeitsbedingungen im Urteil der Beschäftigten halbiert das statistische Risiko, dass keine Gefährdungsanalyse für den Arbeitsplatz vorgenommen wurde. Die Wahrscheinlichkeit für eine Gefährdungsbeurteilung nach Arbeitsschutzgesetz (ArbschG), also einschließlich der Berücksichtigung psychischer Belastungen, liegt bei guten Arbeitsbedingungen um das 1,5-Fache höher als bei den schlecht bewerteten Arbeitsplätzen.?
Die Betrachtung der Ost-West-Differenz macht offenkundig, dass der geschilderte Zusammenhang in gleicher Weise wirkt, im Osten sogar noch stärker ausgeprägt ist als im Westen: D. h. gute Arbeitsbedingungen gehen im Osten mit einer höheren Wahrscheinlichkeit für eine GFB nach ArbSchG als in den westlichen Bundesländern einher. Schlechte Arbeitsbedingungen bei gleichzeitig fehlender GFB lassen sich jedoch ebenfalls überdurchschnittlich häufig in den ostdeutschen Ländern vorfinden (Tabelle 3).

\section{Eine Geschichte der Kontinuität}

Betrachtet man retrospektiv die Entwicklung des AGS in Ostdeutschland hinsichtlich der Formulierung von Ansprüchen und deren realer betrieblicher Umsetzung, so lassen sich grob drei Phasen unterscheiden:

(1) Für die DDR ist eine Gleichzeitigkeit von Ideologieüberschuss und Investitionsrückstand festzustellen. Einerseits gab es im „Arbeiter- und Bauernstaat" strenge AGS-Auflagen, -Vorschriften und -Gesetze, die nach heutiger Einschätzung sogar moderne Präventionsansätze enthielten. Dies führte dazu, dass „,in Vorwegnahme

5 Die Auswertungen der DGB-Index-Daten wurden dankenswerterweise von Tatjana Fuchs durchgeführt. Sie gab auch wichtige Anregungen zum Einfluss der interessenpolitischen Konstellation auf die Qualität der betrieblichen AGS-Politik.

6 Vielen Beschäftigten ist nicht bekannt, ob eine konventionelle GFB durchgeführt wurde, weil sie ausschließlich der Logik von Top-Down-Verfahren folgt (30\% weiß nicht). Es kann nicht verlässlich auf ihre Verbreitung geschlussfolgert werden.

7 Eigene, hier nicht dargestellte Berechnungen von Chi-Quadrat und Odds Ratio. 
Tabelle 3: Arbeitsbedingungen und Verbreitung (in \%) von Gefährdungsbeurteilungen

\begin{tabular}{|c|c|c|c|c|c|c|c|c|}
\hline \multirow[b]{2}{*}{$\begin{array}{l}\text { Arbeitsbedingungen } \\
\text { (DGB-Index) }\end{array}$} & \multicolumn{4}{|c|}{ Westdeutschland } & \multicolumn{4}{|c|}{ Ostdeutschland } \\
\hline & $\begin{array}{l}\text { GFB nach } \\
\text { ArbSchG }\end{array}$ & $\begin{array}{l}\text { konventionelle } \\
\text { GFB }\end{array}$ & Keine GFB & Summe & $\begin{array}{l}\text { GFB nach } \\
\text { ArbSchG }\end{array}$ & $\begin{array}{l}\text { konventionelle } \\
\text { GFB }\end{array}$ & Keine GFB & Summe \\
\hline $\begin{array}{l}\text { schlecht } \\
(0 \text { bis }<50)\end{array}$ & 18,4 & 17,0 & 64,6 & 100,0 & 18,5 & 13,6 & 67,9 & 100,0 \\
\hline $\begin{array}{l}\text { mittel } \\
(50 \text { bis < 80) }\end{array}$ & 25,8 & 17,3 & 56,9 & 100,0 & 30,4 & 14,4 & 55,2 & 100,0 \\
\hline $\begin{array}{l}\text { gut } \\
\text { (80 bis 100) }\end{array}$ & 30,9 & 15,2 & 53,9 & 100,0 & 37,0 & 11,1 & 51,9 & 100,0 \\
\hline \multicolumn{9}{|c|}{$\begin{array}{l}\text { GFB nach ArbSchG - Gefährdungsbeurteilung nach Arbeitsschutzgesetz einschl. Berücksichtigung psychischer Belastungen. } \\
\text { konventionelle GFB - konventionelle Gefährdungsbeurteilung ohne Berücksichtigung psychischer Belastungen. }\end{array}$} \\
\hline Quelle: DGB-Index Gute Arbeit 2 & & & & & \multicolumn{4}{|c|}{ WSI mitteilungen } \\
\hline
\end{tabular}

der Umsetzung der EU-Richtlinien die sicherheitstechnische und arbeitsmedizinische Betreuung im Arbeits- und Gesundheitsschutz schon für kleine Betriebe zur Auflage gemacht werden" (Fröhner 1997, S. 27) konnte. Entsprechend „einem modernen Arbeitsschutzverständnis ..., das sich nicht nur auf die Verhütung von Arbeitsunfällen und Berufskrankheiten konzentriert, sondern auch alle Maßnahmen zur Abwehr arbeitsbedingter Gesundheitsgefahren und zur menschengerechten Gestaltung der Arbeit umfasst", wird diagnostiziert: „In diesem Punkt war das abgelöste DDR-Recht EG-konformer als das bundesdeutsche Recht" (Lehder 1997a, S. 41). Diesem hohen Anspruchsniveau konnten die DDR-Betriebe allerdings aufgrund der Investitionsrückstände in fast allen Betrieben kaum gerecht werden (Windolf et al. 1999). Es gab eigentlich kein Erkenntnis-, sondern ein Arbeitsschutz-Umsetzungsproblem aufgrund einer strukturell „überforderten Volkswirtschaft" (Arendt 2009).

Fehlende Mittel waren der Grund dafür, dass vielerorts kaum Investitionen in den AGS getätigt wurden, nicht nur Maschinen wurden auf „Verschleiß“ gefahren, sondern auch Beschäftigte einseitigen Fehlbelastungen ausgesetzt. Die von vielen Volkseigenen Betrieben angestrebte Würdigung als „Betrieb der ausgezeichneten Qualitätsarbeit" setzte auch einen strengen AGS voraus, der sich aber bei genauerem Hinsehen nicht selten als Fassade entpuppte. In seiner 1988 auch in der DDR erschienenen Reportage „Der Erste“ zitiert Scherzer (1997) den Beschäftigten einer Glashütte, der sich darüber beschwert, dass über Jahre keine technologischen Neuerungen die Produktion erleichtern:

„Vor zwanzig Jahren haben wir alle Flaschen auch noch mit dem Mund geblasen, dann erhielten wir zwei gebrauchte Maschinen aus der BRD (...). Diese Maschinen könnten noch schneller laufen, aber wir können mit unseren Händen nun nicht mehr schneller, bei 1000 Thermogläsern haben wir schon die Schallmauer durchbrochen. Zehn Jahre immer dieselben Handgriffe, erst 600 Kannengläser, dann 650, dann 700... Denn wir wollten mehr verdienen von Jahr zu Jahr. Und um nicht langsamer zu werden, tauschten wir acht in einer Gruppe nie die Arbeitsplätze. Jeder spezialisierte sich auf seine Bewegung. Aber das macht die Arme kaputt und die Gelenke. Ich habe vor 15 Jahren schon vorgeschlagen (...) durch einen Speicher auf der Bühne, die Arbeit zu erleichtern, aber ich habe nie eine Antwort erhalten, nicht mal die 30 Mark Prämie. Das geht dir als Arbeiter dann jahrelang nicht aus der Platte ..." (ebd., S. 69f.).

Festzuhalten bleibt allerdings auch, dass viele Betriebe eine Reihe eigener Einrichtungen der Gesundheitsfürsorge bis hin zu ganzen Polikliniken hatte. Diese Integrationslogik folgte der Idee der betrieblichen Entdifferenzierung, also der Übernahme gesellschaftlicher Aufgaben durch die Betriebe in der DDR.

(2) Die Transformationsphase ist gekennzeichnet von einem betrieblichen und gesellschaftlichen Angleichungsprozess des Ostens an den Westen. Der trennscharfen Ausdifferenzierung der betrieblichen Strukturen durch die Treuhand und ihre Manager folgte in der Regel eine Ausgliederung und Schließung der ,indirekten“ Bereiche. Betriebliche Gesundheitseinrichtungen wurden drastisch zusammengestrichen oder gleich geschlossen.

Gesellschaftlich fand die stufenweise Übernahme des westdeutschen AGSInstitutionensystems statt. Dies vollzog sich in einem betrieblichen Umfeld, das durch die Privatisierungsbestrebungen und Eigentumsübergänge höchst instabil war. Das teilweise bis 1992 noch geltende ostdeutsche AGS-Anspruchsniveau wurde nach und nach abgelöst durch das neue bundesdeutsche Recht. Gesellschaftliche "nachholende Modernisierung" (Zapf 1991) bedeutete also keineswegs, dass die Lücke zwischen Anspruch und Wirklichkeit des AGS aufgehoben wurde, wenngleich sich die Ursachen dafür änderten: So kam es im Prozess der Privatisierung zu einer Neuordnung des Stellenwerts betrieblicher Handlungsfelder. Alle betrieblichen Bereiche, die nicht unmittelbar zum „Kerngeschäft" in der Wertschöpfungskette beitragen konnten, wurden stärker dezimiert als andere. Angesichts eines Beschäftigtenrückgangs auf bis zu ein Zehntel der Vor-Wende-Belegschaft fiel die überdurchschnittliche personelle Schrumpfung der indirekten Bereiche zunächst nicht weiter auf. Ostdeutschland fungierte damit als "Labormaus" des Westens (Brinkmann 2003): Im Großexperiment wurde getestet, wie Unternehmen und Beschäftigte auf den fehlenden Aufbau bzw. die nachhaltige Schwächung des AGS reagieren.

(3) Die Phase der Konsolidierung folgt ab dem Ende der Treuhand Mitte der 1990er Jahre und der allmählichen Durchsetzung der EU-Rahmenrichtlinie für einen präventiven AGS. Schließlich werden in Deutschland die EU-Vorgaben von 1989 erst 1996 in ein nationales Gesetz gegossen. Unterstützt durch eine Reihe von begleitenden Urteilen des Bundesarbeitsgerichts in den Folgejahren ist das Anspruchsniveau seitdem deutlich gestiegen. Die heute beobachtbaren Diskrepanzen sind wie beschrieben zum Teil dem kulturellen Erbe in der Alltagspraxis als nachwirkende Effekte von Transformation und Spätfolgen einiger DDR-Orientierungen geschuldet.

Die Brücke, mit der die Betriebe auch heute die Kluft zu schließen versuchen, lässt sich als Strategie beschreiben, die auf die Inszenierung von AGS-Aktivitäten zur formellen Erfüllung der Vorschriften setzt. 
Dabei sehen sich die aktiven betrieblichen Akteure immer wieder mit dem Kostenargument des Managements konfrontiert, was die innerbetriebliche Durchsetzung von Verbesserungen erheblich erschwert. Hinzu kommt, dass die betriebliche Mobilisierung im Gesundheitsschutz traditionell ein schwieriges Unterfangen darstellt. Die relative Nachrangigkeit dieses Themas - zumal im von Entlassungswellen und Standortschließungen geprägten Osten - wirkt demnach begünstigend auf eine betriebliche Fassadenpolitik, die darauf zielt, die Lücke zwischen AGS-Anspruch und -Wirklichkeit zu verdecken.

\section{1 \\ Kennzeichen des ost- deutschen Sonderwegs}

Eine Charakterisierung des ostdeutschen Sonderwegs lässt sich auf Mikro-, Makround Mesoebene vornehmen - von einem Ausgangspunkt, der entweder in besonderer Weise bis heute nachwirkt oder Zwischenstationen verständlicher macht. So wird das hohe Anspruchsniveau auf der Regulationsebene umgewandelt in einen weniger verbindlichen AGS-Rahmen. Neben weiteren Charakteristika wie dem Planerfüllungspakt der DDR-Zeit und der betrieblichen Notgemeinschaft in der Transformations- und Konsolidierungsphase erklärt das sinkende Regelungsniveau bei gleichzeitig erweiterten manageriellen Entscheidungsspielräumen nach der Wende die Fassadenpolitik im AGS. Und schließlich gibt es auf der Ebene der handelnden Akteure die bis heute nachwirkende DDR-Tradition einer formal zwar vorhandenen, aber faktisch wirkungslosen AGS-Mitbestimmung und einer im Anschluss wahrgenommenen Schwächung der Machtbasis von Beschäftigten und ihren Interessenvertretern, die zu einer Entmündigung ohne echte Teilhabemöglichkeiten führte.

\subsection{HOHE ANSPRÜCHE BEI EINGESCHRÄNKTEN HANDLUNGS- MÖGLICHKEITEN}

Als Ausgangspunkt nach der Wende lässt sich ein grundlegend anderes, von der DDR geprägtes Verständnis von Arbeitsschutz feststellen: Durch Sicherheitsabteilungen in den Betrieben und Kombinaten wur- den die regelmäßig stattfindenden Arbeitsschutzbelehrungen mit den notwendigen Informationen und Materialien versorgt, Kontrollen und Unfallursachenforschung konnten mit (oft aufwendigen) technischen Messungen verbunden werden, das gesetzlich geregelte Neuererwesen - in Westdeutschland vergleichbar mit dem betrieblichen Vorschlagwesen - erstreckte sich zumindest formell auch auf den AGS.

Diese Ausrichtung erforderte einen höheren Personalbestand an haupt- und nebenamtlichen Sicherheitsinspektoren sowie eine enge Verzahnung mit vielen Abteilungen der Arbeitsorganisation. In mancher Hinsicht waren präventive Ansätze ihrer Zeit voraus: So kam es vonseiten der Sicherheitsinspektoren häufig zur Erstellung sogenannter Belastungskataster, die arbeitsplatzbezogen mit den betrieblichen Abteilungen der wissenschaftlichen Arbeitsorganisation entwickelt wurden (Lehder 1997a, S. 35) und mit den Anforderungen einer Gefährdungsbeurteilung nach heutigem Arbeitsschutzgesetz teilweise vergleichbar sind.

\subsection{ERWEITERTE ENTSCHEIDUNGS- SPIELRÄUME BEI SINKENDEM REGULATIONSNIVEAU}

Nach dem Zusammenbruch der DDR wurden auch die AGS-Strukturen neu aus- und eingerichtet und, wie dargelegt, $\mathrm{zu}$ einem überdurchschnittlich großen Teil „verschlankt“. So verblieb z. B. von der 16-Mann-starken Sicherheitsabteilung eines 1.500 Beschäftigte zählenden Maschinenbauunternehmens ${ }^{8}$ eine Sicherheitsfachkraft für die neue Betriebsgröße von 500 Beschäftigten übrig. Dieser Beschäftigte kommt in einem Interview zu dem Resümee: „Der Stellenwert im organisatorischen Arbeitsschutz war in der DDR viel höher. Zum Beispiel wurden monatliche Unterweisungen durchgeführt, also das, was heute höchstens einmal im Jahr stattfindet. Die Durchführung der Unterweisungen war besser, es wurden vergleichsweise mehr Mittel bereitgestellt, es wurde viel mehr Zeit für den Arbeitsschutz aufgebracht."

Kulturell kam es zu einem Bruch durch die Reduzierung der Anforderungen an den betrieblichen AGS und das Aufbrechen des bisher eher symbiotischen Verhältnisses von Gefahrenmessung, -feststellung, -bewertung und Maßnahmenableitung in Kooperation mit staatlichen Kontrolleinrich- tungen und Gewerkschaften. Stattdessen wurden ein Teil dieser Aktivitäten ausgelagert bzw. war jetzt am Markt einzukaufen oder wurde komplett vernachlässigt.

Das Verhältnis von Staat und Betrieb gestaltete sich nunmehr durch die Gewerbeaufsichtsämter nach westdeutschem Vorbild, d. h. in der Tendenz eher restriktiv und sanktionsorientiert als partnerschaftlich. Gleichzeitig ließen die Qualität und Quantität von Kontrollen deutlich nach, was auch zur Folge hatte, dass Unternehmensakteure die gesetzlichen AGS-Anforderungen in diesem Bereich als weniger relevant einschätzen: „Jetzt wird vieles in die Verantwortung des Arbeitgebers gelegt. Wenn es um das Leben der Leute geht, sollte man aber schon klare Grenzwerte haben, also dass man klar macht ,bis hierin und nicht weiter dürft ihr gehen'. Das kümmert heute aber keinen. Das war zu DDR-Zeiten anders", so die bereits zitierte Sicherheitsfachkraft.

Der weitgehend „kritiklosen Übernahme des Arbeitsschutzsystems der alten Bundesländer" (Lehder 1997b, S. 88) kam auch entgegen, dass der Ermessensspielraum für die Unternehmen innerhalb des neuen Regelungswerkes stieg, was angesichts der breiten Bedrohung der Unternehmensbestände eher zu einer Vernachlässigung des AGS-Feldes führte. Im Zuge der anstehenden Herausforderungen für Unternehmen - Umstrukturierungen, Neuausrichtung auf den Märkten, Herstellung von Wettbewerbsfähigkeit und operativer Flexibilität - gerieten in den 1990er Jahren Strategien der internen Organisationsentwicklung, der Pflege des Personalbestands und der arbeitssicherheitlichen und gesundheitlichen Fürsorge für die Beschäftigten in die Defensive.

Das Management vieler ostdeutscher Betriebe sah Erfolg ausschließlich in der Steigerung von Produktivität. Für die Beschäftigten und ihre Interessenvertreter (soweit vorhanden) bemaß sich der Erfolg zunächst am Unternehmensfortbestand und später am Abbau der Lohndiskrepanz zum Westen. Diese Anreizstruktur führte dazu, dass über die letzten 20 Jahre beide Seiten ein eher stiefmütterliches Verhältnis zur AGS-Praxis aufrechterhielten. Die Spätfolgen dieses Experiments zeigen sich

8 Ein Fallbetrieb im PARGEMA-Projekt, zur Einordnung vgl. den Projektabschlussbericht (Erscheint bei edition sigma 2010) 
bis heute in Form eines defizienten, eingeschränkt gesetzeskonformen AGS.

\subsection{PLANERFÜLLUNGSPAKT UND BETRIEBLICHE NOTGEMEINSCHAFT}

Die kontinuierliche Diskrepanz zwischen Anspruch und Wirklichkeit wird in der DDR beispielsweise mit Blick auf die ökologischen Bedingungen in der Industrie (mit massiven Ausstrahlungseffekten in die Regionen) manifest. Neben den bereits genannten war eine der Ursachen dafür „die isolierte Handhabung des Arbeitsschutzes, abgekoppelt von der Wirtschaftspolitik des Unternehmens" (Lehder 1997a, S. 34; vgl. auch Kutzsche et al. 1993). Der AGS-Stellenwert innerhalb der betriebspolitischen Handlungsfelder konnte von Betrieb zu Betrieb stark variieren, der „stark reglementierende Charakter des Vorschriftenwerkes der DDR“ (Lehder 1997b, S. 88) reduzierte allerdings auch die Ermessensspielräume.

Hilfreich dafür, dass Umwelt- und auch die AGS-Probleme nicht zu offenkundig in Betriebskollektiv und Öffentlichkeit diskutiert wurden, war die normative Bindung des Planerfüllungspaktes. Geschäftsleitung und Belegschaft bedienten einmütig die planwirtschaftlichen Vorgaben der zentralen Lenkungsgremien in Berlin oder den Bezirkshauptstädten mit den gewünschten Kennziffern und konnten im Gegenzug mit der Lieferung von benötigten Rohstoffen und anderen Ressourcen rechnen.

Nach der Wende führte die massive Personalentlassung bei den verbliebenen Beschäftigten einerseits zu einer erhöhten Hinnahmebereitschaft schlechter Arbeitsbedingungen. Andererseits sorgte die Privatisierung als Versuch, den Fortbestand des Betriebs zu sichern, für die gemeinsame Wahrnehmung von Beschäftigten und Management als betriebliche Notgemeinschaft. Ihr Wille zur Rettung und Konsolidierung des Unternehmens ließ Fragen nach den Arbeitsbedingungen genauso wie AGS-Fragen weit in den Hintergrund treten.

\subsection{FASSADENPOLITIK ALS FORTSETZUNG DER DDR- UND TRANSFORMATIONSPRAXIS}

Durch die in der DDR einschlägigen arbeitsplatzbezogenen Belastungskataster waren die betrieblichen AGS-Akteure mit der Herangehensweise bereits vertraut,
Risiken arbeitsplatzbezogen zu bewerten. Viele befragte Sicherheitsfachkräfte sehen in der Gefährdungsbeurteilung daher ein Instrument, das der gleichen Logik folgt, ohne dass die Neuerungen (Gebote zur Erfassung psychischer Belastungen und zur Beteiligung von Beschäftigten) überhaupt zur Kenntnis genommen werden. Die Verbreitung von Gefährdungsbeurteilungen erklärt sich daher aus der Vertrautheit mit diesem Ansatz, aber auch aus der Möglichkeit, problematische Arbeitsbedingungen dethematisieren oder schönen zu können.

Darauf weisen Aussagen wie die folgende einer Sicherheitsfachkraft im Interview hin: „Schönfärberei machen wir doch heute genauso wie damals. Nehmen Sie die Gefährdungsanalyse, da wird ein enormer Schreibaufwand betrieben, für den Fall, dass mal jemand nachfragt. An dem, was da im Betrieb abläuft, ändert sich aber nichts. Da wird, kurz bevor die BG kommt, noch mal ordentlich gelüftet und schon sinkt die Feinstaubbelastung“" Auch aus Interviews mit Betriebsbetreuern der Gewerbeaufsicht für Ostdeutschland ist bekannt, dass die Erscheinungsweise der Gefährdungsbeurteilung stark differieren kann: „Nicht überall, wo Gefährdungsbeurteilung draufsteht, steckt auch Gefährdungsbeurteilung drin!", wie ein Aufsichtsbeamter und Kenner des AGS insbesondere im verarbeitenden Gewerbe zu Protokoll gibt.

\subsection{RESIGNATION DURCH KANALISIERTE MITBESTIMMUNG}

Eine Besonderheit war die Einbindung der Einheitsgewerkschaft FDGB (neben den staatlichen Organen) in die Kontrolle des Arbeitsschutzes. Damit verbunden war das Recht der Arbeitsschutzinspektion, die beim FDGB angesiedelt war, Auflagen an Betriebsleiter zu erteilen, „Arbeitsstätten, Betriebsanlagen und -einrichtungen jederzeit zu betreten, Einsicht in Unterlagen zu nehmen und Auskünfte zu verlangen. Sie konnte ferner Ursachen von Gefährdungen, von Arbeitsunfällen, Berufskrankheiten und sonstigen arbeitsbedingten Erkrankungen in den Betrieben ermitteln " (Lehder 1997a, S. 38). Auch wenn die Gewerkschaften in der DDR mit Blick auf ihr Verhältnis zur SED kaum als eigenständiger, emanzipierter Akteur angesehen werden können, so sind doch ihre Machtmöglichkeiten gegenüber den Betriebslei- tungen insbesondere in Gesundheitsfragen nicht zu unterschätzen.

Des Weiteren unterlagen Verbesserungen im AGS wie alle anderen staatlich gewünschten Initiativen von Beschäftigtenseite dem Neuererwesen, das über entsprechende Anreizstrukturen darauf zielte, Verbesserungsideen aufzunehmen. Neben seiner Rolle als „systemkonforme ,Beschäftigungstherapie “" (Hartmann 1988, S. 16) und als Konfliktabsorber (Ortlieb/Stein 2008, S. 397) zielte die gesetzlich geregelte Neuererbewegung in erster Linie auf Rationalisierungsmaßnahmen ab und diente nicht vorrangig der Schaffung entlastender Arbeitsbedingungen.

Diese Formen der kanalisierten Mitbestimmung veränderten im Prozess der Anerkennung durch Neuererkommissionen oder durch den FDGB häufig den Charakter der ursprünglichen Ideen. Schließlich führte die Belobigung einer Idee häufig zum Stillhalten bei den Beschäftigten, auch wenn diese nicht zur Umsetzung kam. Viele Beschäftigte und Interessenvertreter resignierten und hielten sich in der Folge zurück mit ihrem Engagement und einem Überschwang an Verbesserungsvorschlägen.

\subsection{SCHWACHE MACHTBASIS FÜR BESCHÄFTIGTENINTERESSEN}

Den Belegschaftsvertretungen in Ostdeutschland fehlt heute die Machtbasis für die Kontrolle, Verbesserung oder Durchsetzung höherer AGS-Standards. Der große Teil von Unternehmen ohne irgendeine Interessenvertretung verunmöglicht zumeist Ansätze beteiligungsorientierter Erneuerung des AGS, wie es z. B. das Arbeitsschutzgesetz vorsieht.

Zurückzuführen ist diese Schwäche an arbeitsmarktbedingter Primärmacht und kollektiver Sekundärmacht auf den spezifischen Entwicklungspfad, den Ostdeutschland im Transformationsverlauf genommen hat. Der Institutionentransfer und der damit verbundene rasche Strukturaufbau und -umbau wurde formell zwar vollzogen. Die jeweiligen Institutionen wie Tarifverträge, Arbeitgeberverbände, Gewerkschaften, Betriebsverfassungsgesetz, Gewerbeaufsichtsämter, Berufsgenossenschaften u. a. waren jedoch mit deutlich anderen Rahmenbedingungen konfrontiert, als sie sich im westdeutschen Feld etabliert und entwickelt hatten. Die Arbeitsmarktsituation verhinderte die Wahrnehmung von 
Exit- und Voice-Optionen gleichermaßen, schwächte also die betriebliche Machtposition von Beschäftigten erheblich.

Trotz der breiten Varianz der Interaktionsbeziehungen zwischen Management und Betriebsrat lässt sich mit MüllerJentsch (1997) tendenziell eine Beteiligung des Betriebsrats an Managementaufgaben beobachten. Der im Sinne eines „Co-Managers“ agierende Betriebsrat übernimmt Aufgaben der Modernisierung und Restrukturierung der Arbeits- und Unternehmensorganisation. Die Funktion bzw. das Selbstverständnis vieler ostdeutscher Betriebsräte in den 1990er Jahren ist mit denen des Co-Managers vergleichbar: Die Restrukturierung der ostdeutschen Betriebe mit all ihren personalpolitischen Konsequenzen schuf eine Atmosphäre „notgemeinschaftlicher Kooperation“, die durch das gemeinsame Grundinteresse am Erhalt des Betriebs gekennzeichnet war (Artus 2001; Behr/Engel 2001). Vor diesem Hintergrund wurden die Personalentlassungswellen, die mit dem Auslaufen der Kündigungsschutzregelung Mitte 1991 einhergingen, von den ostdeutschen Betriebsräten akzeptiert. Sie konzentrierten sich auf die soziale Abfederung der Entlassungen, wodurch sie zunehmend die Rolle von Sozialmanagern übernahmen (Röbenack 2005, S. 150). Im Vergleich zu ihrem westdeutschen Pendant gelten ostdeutsche Betriebsräte daher eher als unpolitischer und sachlich-pragmatischer (vgl. Kädtler/ Kottwitz 1994, S. 27).

Für die in der WSI-Befragung abgebildeten Betriebe in Ostdeutschland kann deshalb festgehalten werden, dass die beschriebenen AGS-Routinen überwiegend in ihrer konventionellen Ausprägung (als Gefährdungsbeurteilung klassischer Belastungen) vorkommen, weil die Interessenvertreter in diesem Bereich keine Vorstöße gegen den Willen von Geschäftsführung oder Sicherheitsfachkraft wagten, geschweige denn erstritten oder erkämpft haben. Nach der Konsolidierungsphase für die Mitbestimmungsstrukturen in den Betriebsratsbetrieben könnte es aber zu einem veränderten Selbstverständnis kommen. Die PARGEMA-Projektbetriebe geben Hinweise auf eine „Renaissance“ des AGS-Themas, dessen sich jetzt zunehmend die Interessenvertreter annehmen.

Ostdeutsche Betriebsräte werden heute (nach eigenem Bekunden in der WSIBetriebsrätebefragung) weniger in den GFB-Prozess einbezogen (50 \%) als ihre
Kollegen im Westen (56\%), was insbesondere die Ermittlung von Gefährdungen und Belastungen betrifft (Unterschied für diesen Teilaspekt rund 10-\%-Punkte). Für Ostdeutschland sehen wir in der Breite eine eher pragmatisch geprägte Herangehensweise an die GFB, die formal durchgeführt, aber kaum als der grundlegende AGS-Ansatz im Betrieb mit Berücksichtigung psychischer Belastungen und Beteiligungsanspruch genutzt wird.

Mit Blick auf die Betriebsratsdichte kann für Ostdeutschland festgehalten werden, dass die interessenpolitische Flankierung bzw. Durchsetzung von AGS nicht gegeben ist. Die Politisierbarkeit von Belegschaftsanliegen und der Wille zum Austragen von Konflikten ist durch die nachwirkende Notgemeinschaftsprägung immer noch schwach, auch wenn sich hier bereits verschiedene Betriebs- bzw. Belegschaftstypen im Umgang mit der Erosion des Nachwendepaktes kennzeichnen lassen (Behr et al. 2008).

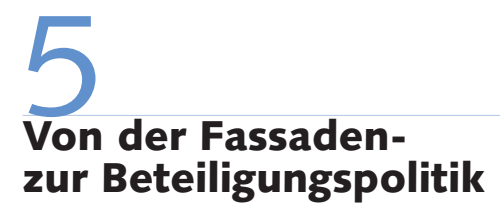

Faktisch haben wir es in Ostdeutschland aufgrund der Machtverhältnisse und der dominanten Co-Management-Strategie von Betriebsräten mit weitgehend entmündigten Beschäftigten ohne Teilhabemöglichkeiten zu tun. Die Umsetzung partizipativer Ansprüche allgemein und Beteiligungsangebote im AGS im Besonderen ist eher rar gesät.

Die Ausführungen zum AGS in Ostdeutschland zeigen die Prägekraft kultureller Pfade. So erweist sich die Diskrepanz zwischen Anspruch und Wirklichkeit in der DDR, der Transformationsphase und bis heute als beständiges Merkmal einer weit verbreiteten AGS-Praxis. Diesem Sachverhalt muss, wenn es um die Bewertung und Weiterentwicklung praxisrelevanter AGS-Standards geht, Rechnung getragen werden. Die Handlungsfähigkeit derer müsste gestärkt werden, die AGSFragen nachhaltig bearbeiten wollen. Neben den gängigen, leicht greifbaren Möglichkeiten (bessere Qualifizierung, Bereitstellen personeller Ressourcen etc.) bedarf es dafür einer stärkeren Sensibilisierung für die gesellschaftliche Relevanz des Themas Gesundheit. Der massive Anstieg psychischer Erkrankungen infolge ständig wachsender Leistungsanforderungen bietet hierfür einen wichtigen Anknüpfungspunkt.

Auf der betrieblichen Ebene kann die Aufwertung dieses Themas unter anderem dadurch gelingen, dass man die Beschäftigten an der Verbesserung der AGS-Praxis beteiligt. Ein geeignetes Instrument dafür ist die Gefährdungsbeurteilung, bei der Beschäftigte an allen relevanten Prozessen - sowohl bei der Erfassung und Bewertung von Belastungen als auch bei deren Minimierung - aktiv teilnehmen sollten. Betriebliche Beispiele zeigen, dass diese Beteiligungsstrategie nicht nur die Gesundheitsschutzfassade bröckeln oder ganz zum Einsturz bringen lässt, sondern auch die Chance für den Aufbau eines wirksamen und nachhaltigen Gesundheitsmanagements in sich birgt. Die nunmehr weitgehende Konsistenz von Anspruch und Wirklichkeit in diesen Betrieben verhalf den betrieblichen AGS-Akteuren zu mehr Durchsetzungsmacht (Becker et al. 2010b).

Best-Practice-Fälle wie diese haben einen Nachahmungseffekt, der nicht unterschätzt werden sollte. Der Transfer von Erfahrungen betrieblicher Gesundheitsschutzprojekte, bei dem Schwierigkeiten und Hürden nicht ausgespart werden, bestärkt viele Interessenvertreter, sich des Themas anzunehmen und mit der Initiierung einer Gefährdungsbeurteilung in die Offensive zu gehen.

In diesem Kontext müssen wiederum kulturelle und regionale Spezifika berücksichtigt werden. So kann es für die strukturell schwächeren ostdeutschen Betriebsräte zielführend sein, niedrigschwellige Einstiegsszenarien in einer Gefährdungsbeurteilung zu wählen, an deren Anfang nicht zwangsläufig eine Betriebsvereinbarung stehen muss.

Für Ost wie West gilt, dass es offenbar unabdingbar ist, Kontrollen durch die Gewerbeaufsichtsämter zu verstärken und Vergehen leichter zu sanktionieren. Es muss daher auf einen gesellschaftlichen Konsens hingewirkt werden, Verstöße gegen die gesetzlichen Rahmenbedingungen nicht als Kavaliersdelikte zu behandeln. Auch auf der betrieblichen Ebene muss klar sein, dass die Folgen von Gesundheitsgefährdungen betriebswirtschaftlich nicht wie externe Effekte behandelt werden dürfen - wie Kosten also, die auf die Gesellschaft abgewälzt werden können. 
Ahlers, E./Brussig, M. (2005): Gefährdungsbeurteilungen in der betrieblichen Praxis, in: WSI Mitteilungen 9, S. 517-523

Ahrendt, M. (2009): Die Wissenschaftliche Arbeitsorganisation (WAO) als betriebliches Aufgabengebiet in der DDR - eine Rückschau, in: Zeitschrift für Arbeitswissenschaft 2, S. 157-159

Artus, I. (2001): Die Krise des deutschen Tarifsystems. Die Erosion des Flächentarifvertrags in Ost und West, Wiesbaden

Beck, D./Lenhardt, U. (2009): Verbreitung der Gefährdungsbeurteilung in Deutschland, in: Prävention und Gesundheitsförderung 4, S. 71-76

Becker, K./Brinkmann, U./Engel, T. (2010a): Beteiligung in der Gefährdungsbeurteilung - Der Weg zu einem nachhaltigen Gesundheitsmanagement, in: Schröder, L./Urban, H.J. (Hrsg.): Gute Arbeit. Handlungsfelder für Betriebe, Politik, Gewerkschaften, Frankfurt a.M., S. 282-297

Becker, K./Brinkmann, U./Engel, T. (2010b): Gefährdungsbeurteilungen als Präventionsspiralen zur Gestaltung von Arbeit, in: Kratzer, N. (Hrsg.): Partizipatives Gesundheitsmanagement. Leistungspolitik Gesundheit - Beteiligung, (im Erscheinen)

Behr, M./Engel, T. (2001): Entwicklungsverläufe und Entwicklungsszenarien ostdeutscher Personalpolitik, in: Lang, R. (Hrsg.): Zehn Jahre Personalarbeit in den neuen Bundesländern, München und Mering, S. $255-278$

Behr, M./Engel, T./Hinz, A. (2008): Blockierte Modernisierung ostdeutscher Unternehmenskulturen als Standortrisiko, in: Benthin, R./ Brinkmann, U. (Hrsg.): Unternehmenskultur und Mitbestimmung. Betriebliche Integration zwischen Konsens und Konflikt, Frankfurt a. M./ New York, S. 259-282

Brenke, K./Zimmermann, K.F. (2009): Ostdeutschland 20 Jahre nach dem Mauerfall, Vierteljahrshefte zur Wirtschaftsforschung/Quarterly Journal of Economic Research 2, S. 32-62

Brinkmann, U. (2002): Umbruch von unten? Betriebliche Akteure in der ostdeutschen Transformation, München/Mering

Brinkmann, U. (2003): Die Labormaus des Westens: Ostdeutschland als Vorwegnahme des Neuen Produktionsmodells?, in: Dörre, K./Röttger, B. (Hrsg.): Das neue Marktregime, Hamburg, S. 250-269

Fröhner, K.-D. (Hrsg.) (1997): Sicherheit und Gesundheit im Betrieb. Deutsch-deutsche Transformation und europäischer Wandel, KSPW: Transformationsprozesse, Band 15, Opladen

Fröhner, K.-D. (1997): Sicherheit und Gesundheit als interdisziplinäres Handlungsfeld, in: Ders. (Hrsg.): a.a.O., S. 13-28

Fuchs, T. (2007): Wie nachhaltig ist die Arbeitswelt in Deutschland?, Stadtbergen

Görzig, B./Gornig, M./Werwatz, A. (2005): Nichtparametrische Dekomposition der Lohndifferenzen zwischen ost-und westdeutschen Betrieben. Allgemeines Statistisches Archiv 3, S. 365-381
Hartmann, M. (1988): Die Neuererbewegung. Das betriebliche Vorschlagswesen in der DDR, Köln

Heyde, K./Macco, K. (2010): Krankheitsbedingte Fehlzeiten aufgrund psychischer Erkrankungen, in: Badura, B. (Hrsg.): Fehlzeiten-Report 2009, Berlin, S. 31-40

Kädtler, J./Kottwitz, G. (1994): Industrielle Beziehungen in Ostdeutschland, in: Industrielle Beziehungen 1, S. 13-38

Kocyba, H./Voswinkel, S. (2007): Krankheitsverleugnung - Das Janusgesicht sinkender Fehlzeiten, in: WSI Mitteilungen 3, S. 131-137

Kutzsche, U./Lehder, G./Peaech, H./Wienhold, L. (1993): Probleme der Sicherstellung sicherheitstechnischer und arbeitsmedizinischer Betreuung in den neuen Bundesländern, Schriftenreihe der Bundesanstalt für Arbeitsschutz, Fb 684, Bremerhaven

Lehder, G. (1997a): Der Umbruch in den formellen Bedingungen, in: Fröhner, K.D. (Hrsg.): Sicherheit und Gesundheit im Betrieb. Deutschdeutsche Transformation und europäischer Wandel, KSPW: Transformationsprozesse, Band 15, Opladen, S. 29-42

Lehder, G. (1997b): Der Zustand des neu geordneten Arbeitsschutzes, in: Fröhner, K.D. (Hrsg.): Sicherheit und Gesundheit im Betrieb. Deutsch-deutsche Transformation und europäischer Wandel, KSPW: Transformationsprozesse, Band 15, Opladen, S. 88-100

Lenhardt, U./Kuhn, J./Reusch, J. (2010): Die Arbeitswelt von heute. Daten, Schwerpunkte, Trends, in: Schröder, L./Urban, H.J. (Hrsg.): Gute Arbeit. Handlungsfelder für Betriebe, Politik, Gewerkschaften, Frankfurt a.M., S. 432-491

Müller-Jentsch, W. (1997): Soziologie der Industriellen Beziehungen. Eine Einführung, Frankfurt/Main, New York.

Offe, C. (1994): Der Tunnel am Ende des Lichts. Erkundungen der politischen Transformation im Neuen Osten, Frankfurt a.M.

Ortlieb, R./Stein, S. (2008): „Ideen kann man nicht wie Kirschen vom Baum pflücken“. Eine politikorientierte Analyse des Betrieblichen Vorschlagswesens mit Fallbeispiel, in: Zeitschrift für Personalforschung 4, S. $388-412$

Röbenack, S. (2005): „Aber meistens einfach nur ein Kollege“. Über die ersten Betriebsräte in Ostdeutschland, München

Scherzer, L. (1997): Der Erste. Mit einem weiterführenden Bericht „Der letzte Erste" ${ }^{\prime}$, Berlin

Siegrist, J./Dragano, N. (2008): Psychosoziale Belastungen und Erkrankungsrisiken im Erwerbsleben. Bundesgesundheitsblatt - Gesundheitsforschung - Gesundheitsschutz 3, S. 305-312

Windolf, P./Brinkmann, U./Kulke, D. (1999): Warum blüht der Osten nicht? Zur Transformation der ostdeutschen Betriebe, Berlin

Zapf, W. (1991): Der Untergang der DDR und die soziologische Theorie der Modernisierung, in: Giesen, B./Leggewie, C. (Hrsg.): Experiment Vereinigung. Ein sozialer Großversuch, Berlin, S. 38-51 